\title{
Actividad física, salud y funcionalidad entre adultos $\triangleq$ mayores según la localidad geográfica
}

\author{
Atividade física, saúde e funcionalidade entre idosos de acordo com a localização \\ geográfica
}

\section{AUTHOR'S \\ Daniel Vicentini de Oliveira ${ }^{1}$ (D) \\ Walter Aquiles Sepúlveda Loyola² (D) \\ Gabriel Lucas Morais Freire ${ }^{3}$ (D) \\ Renata Pires Tricanico Maciel $^{2}$ (D) \\ Vera Lúcia Kerber ${ }^{1}$ (D) \\ José Roberto Andrade do Nascimento \\ Júnior $^{3}$ iD \\ 1 Universidade Cesumar, Departamento de Pós- graduação Stricto Sensu em Promoção da Saúde, \\ Maringá, Paraná, Brasil. \\ 2 Universidade Estadual de Londrina. \\ Departamento de Pós-graduação Stricto Sensu em Ciências da Reabilitaçāo, Londrina, Paraná, Brasil. 3 Universidade Federal do Vale do São Francisco. Departamento de Pós-graduação Stricto Sensu em Educação Física, Petrolina, Pernambuco, Brasil.}

\section{CORRESPONDING}

Daniel Vicentini de Oliveira

d.vicentini@hotmail.com

Avenida Guedner, 1610. Maringá, Paraná,

Brasil.

CEP: 87050-900.

DOI

10.12820/rbafs.24e0109

\section{(cC) BY-NC-SA}

This work is licensed under the Creative Commons Attribution-NonCommercial-ShareAlike 4.0 International License

\begin{abstract}
RESUMEN
Este estudio transversal rcomparó las diferencias sociodemográficas, auto percepción de salud, nivel de actividad física y la funcionalidad de los adultos mayores adscritos a centros de atención pública de diferentes sectores geográficos de una ciudad brasileira. Estudio epidemiológico con 654 adultos mayores (69,42 \pm 7,51 años), de ambos los sexos, de la comuna de Maringá, Brasil. Los sujetos fueron reclutados de cuatro diferentes regiones. Fue evaluado el perfil sociodemográfico, nivel educacional, auto percepción de salud, nivel de actividad física y la funcionalidad. Los ancianos entre $60-69$ y 70 - 79 años tenían tres veces más probabilidades de vivir en las regiones sur y oeste. Las personas mayores con educación superior tienen más probabilidades de vivir en el este y oeste. Las personas mayores con mala percepción de salud y que informaron tomar más de dos medicamentos tenían más del 50,0\% de probabilidades de vivir en la región oriental. La prevalencia del uso de más de dos medicamentos es mayor en el sector oeste $(51,0 \%)$ y sur $(52,0 \%)$. Los adultos mayores del sector norte realizan menos tiempo de actividad física moreda y vigorosa ( $\mathrm{p}=0.001)$, y los adultos mayores de la región sur presentan menor tiempo sentado $(p=0.001)$. Los adultos mayores de la región norte presentaban mejor funcionalidad $(\mathrm{p}=0.001)$. Existen diferencias entre los factores sociodemográficos, condición de salud, nivel de actividad física, funcionalidad y participación social entre los adultos mayores que viven en diferentes barrios de una comuna que deben ser consideradas al momento de crear estrategias de salud pública.
\end{abstract}

Keywords: Envejecimiento; Sistema de salud; Población; Actividad física.

\section{RESUMO}

Este estudo teve como objetivo comparar as diferenças sociodemográficas, percepção de saúde, nível de atividade física e funcionalidade de idosos alocados em centros públicos de atenção em saúde, em diferentes setores geográficos de uma cidade brasileira. Estudo epidemiológico, transversal, com 654 idosos (69,42 \pm 7,51 anos de idade), de ambos os sexos, da cidade de Maringá, Brasil. Os indivíduos foram recrutados em quatro regiōes diferentes. Foram avaliados o perfil sociodemográfico, escolaridade, autopercep̧ão de saúde, nível de atividade física e funcionalidade Os idosos entre 60-69 e 70-79 anos tiveram três vezes mais chances de morarem nas regiôes sul e oeste. Os idosos com ensino superior têm maior probabilidade de morarem no leste e oeste. As pessoas idosas com baixa percepção de saúde que relataram tomar mais de dois medicamentos tiveram mais de 50,0\% de chance de morarem na região leste. A prevalência do uso de mais de duas drogas é maior no setor ocidental $(51,0 \%)$ e no setor sul $(52,0 \%)$. Os idosos no setor norte têm menos tempo para atividade física vigorosa e mais vigorosa $(p=0,001)$, e os idosos na região sul têm menos tempo sentado $(p=0,001)$. Existem diferenças entre fatores sociodemográficos, condição de saúde, nível de atividade física, funcionalidade e participação social entre idosos residentes em diferentes bairros de uma comunidade que devem ser considerados na criação de estratégias de saúde pública nos diferentes setores.

Palavras-chave: Envelhecimento; Sistema saúde; População; Atividade física.

\section{Introducción}

$\mathrm{El}$ proceso de envejecimiento engloba varios cambios que están directamente relacionados con el estilo de vida, condiciones socioeconómicas y, en algunos casos, enfermedades crónicas que afectan la salud del individuo $^{1}$. Dentro de los factores relacionados al estilo de vida, la inactividad física, es uno de los factores de ries- go que contribuye al desarrollo de múltiples morbilidades, aumentando la probabilidad de hospitalizaciones, tiempo de internación y de readmisión, así como los costos de salud, reduce la calidad de vida y aumenta la dependencia y la mortalidad ${ }^{2}$.

En Brasil, la población está envejeciendo a un ritmo acelerado. Los resultados de investigaciones realizadas 
por la Organización Mundial de la Salud demuestran un aumento importante de la población de más de 60 años de edad para las próximas décadas ${ }^{3}$. Esta es una tendencia que continuará durante los próximos años, considerando que en el año 2025 habrán más de 800 millones de personas mayores de 65 años en todo el mundo. De acuerdo con la Organización de las Naciones Unidas se prevé un margen de 1.100 .000 de adultos mayores para el año 2025, mientras que en el año 2050 en todo el mundo el número de adultos mayores habrá superado el número de jóvenes ${ }^{4}$.

Estrategias para promover estilos de vida saludable son necesarias para hacer que este fenómeno demográfico sea más sustentable ${ }^{1}$. La evaluación de diferentes niveles de limitaciones funcionales es fundamental para entender mejor las diferencias entre las necesidades y demandas de los adultos mayores, pues los niveles de dependencia están asociados a una mayor demanda por cuidados a largo plazo y mortalidad ${ }^{5}$. Por otro lado, estudios sugieren que el análisis de diferentes regiones, aspectos socioeconómicos y demográficos son de gran importancia para la planificación y el delineamiento de políticas públicas orientadas al bienestar de la población adulta mayor ${ }^{6,7}$. Sin embargo, es necesaria más evidencia para conocer las diferencias asociadas a la salud y la funcionalidad que pueden existir dependiendo de la localidad donde un adulto mayor vive. Según la Organización Mundial de la Salud la localidad es un factor contextual importante para la salud de una persona ${ }^{8}$.

El objetivo de este estudio fue comparar las diferencias en las características sociodemográficas, nivel de actividad física y la funcionalidad de los adultos mayores adcritos a centros de atención pública de diferentes sectores geográficos de una ciudad.

\section{Métodos}

Se condujo un estudio epidemiológico descriptivo, de corte transversal con 654 adultos mayores de la comuna de Maringá, Paraná, Brasil. Los sujetos fueron reclutados, de los centros de atención primaria (CAP) del sistema público de la ciudad, los cuales fueron divididos en cuatro diferentes regiones: región Este (7 CAPs) que contempla $21,8 \%$ de la población, región norte ( 9 CAPs) que contempla $34,5 \%$ de la población, región oeste (8 CAPs) que contempla $23,2 \%$ de la población y región sur (8 CAPs) que contempla 20,4\% de la población. El cálculo del tamaño de la muestra fue obtenido a través del software StatDisk versión 8.4, considerando una población de 42.258 adultos mayores según los datos de la Secretaria de Salud del Municipio de Maringá, del año 2016. Los centros de atención primaria evaluados fueron seleccionados aleatoriamente.

Fueron incluidos individuos con edad mayor o igual a 60 años, capacidad auditiva y cognitiva conservada evaluada por el cuestionario Mini Mental, utilizando el punto de corte de Brucki, Nitrini, Caramelli, Bertolucci, y Okamoto9. Individuos con dificultad auditiva severa, o una puntuación del Mini Mental bajo lo recomendado de acuerdo a su nivel educacional fueron excluidos del estudio. La participación de los adultos mayores en el estudio fue voluntaria y ellos tuvieron que firmar un consentimiento informado.

Las evaluaciones fueron realizadas por investigadores previamente capacitados. Todos los cuestionarios y evaluaciones fueron realizados de manera presencial, mañana y tarde, según disponibilidad de los autores, en una entrevista privada con cada adulto mayor, con el investigador responsable. Cada entrevista duró, en promedio, 20 minutos por persona mayor.

Para la caracterización del perfil sociodemográfico, fue realizado un cuestionario estructurado, clasificando a los individuos según su edad, género, estado civil, raza, tipo de renta (activa o pasiva), salario mensual en Salario Mínimo (SM) usando referencia del censo del año 2016 del Instituto Brasilero de Estadística (IBGE) (1-2 SM; 2-3 SM; superior a $3 \mathrm{SM}$ ), jubilación, nivel educacional e historia de tabaquismo. Además, en este cuestionario inicial se evaluó la autopercepción de la salud (mala, regular, buena o muy buena), el histórico de caídas en los últimos seis meses, cantidad de medicamentos y número de morbilidades.

La discapacidad y salud fue evaluada por medio del World Health Organization Disability Assessment Schedule (WHODAS 2.0) ${ }^{10}$, que cuenta de 12 Ítems distribuidos en seis dominios: comprensión y comunicación (CC), movilidad (MB), autocuidado (AC), relaciones interpersonales (RI), actividad de la vida diaria (AVD) y participación social (PS). La escala varia de 0 puntos (ninguna dificultad) hasta 4 puntos (extrema dificultad $)^{11}$.

El nivel de actividad física de los adultos mayores fue evaluado utilizándose la versión abreviada del Cuestionario Internacional de Actividad Física (IPAQ) ${ }^{12}$. E1 cual está formado por siete preguntas abiertas que permiten estimar el tiempo utilizado por semana en diferentes tipos de actividades (caminata y esfuerzos físicos de intensidades moderados y vigorosos) y de inactividad (posición sentada). El nivel de actividad física fue 
clasificado en sedentario, irregularmente activo, activo y muy activo. Se consideró el tiempo de 150 minutos por semana de actividades físicas para que el sujeto se clasifique como físicamente activo; durante menos de diez minutos a la semana, el sujeto se consideró sedentario y aquellos que realizaron al menos diez minutos, pero no alcanzaron los 150 minutos, se consideraron insuficientemente activos ${ }^{12}$.

$\mathrm{E} 1$ análisis de los datos fue realizado por medio del Software SPSS versión 22.0. Se utilizaron frecuencias y porcentajes como medidas descriptivas para las variables categóricas. El test de normalidad Kolmogorov-Smirnov fue realizado para probar la normalidad de los datos. Como los datos presentaron una distribución no paramétrica, los resultados fueron expresados en mediana $(\mathrm{Md})$ y sus cuartiles (Q1-Q3). El test de Chi-cuadrado fue usado para investigar las diferencias entre las variables categóricas. El análisis de regresión logística multivariable con un ajuste robusto de la varianza, total y ajustado, también se utilizó para estimar las tasas de prevalencia y los intervalos de confianza del 95\%. Para modelar el análisis de regresión logística, solo se consideraron las variables con un nivel de significancia igual o inferior a 0,20 para la asociación en la prueba de Chi-cuadrado. En el análisis ajustado, todas las variables se incluyeron en el modelo, independientemente del valor $\mathrm{p}$ del análisis total.

El test Kruscal-Wallis y test Mann-Whitney para diferencias entre las variables numéricas, de los diferentes sectores. Se consideró un nivel de significancia de $\mathrm{p}<0.05$.

\section{Resultados}

Fueron evaluados 654 adultos mayores, observándose una mayor prevalencia de mujeres $(56,0 \%)$, personas casadas (61,3\%), y un rango etario entre 60 a 69 años (59,0\%). E1 75,0\% de los sujetos eran jubilados y el $70,0 \%$ tenían una renta mensual de entre 1 a 2 salarios mínimos. El 43,0\% de los adultos mayores no tenía la enseñanza básica completa (Tabla 1).

Al comparar a los adultos mayores de las cuatro regiones geográficas de la ciudad (norte, oeste, este y sur), se observaron diferencias entre ellas en el rango etario $(p=0.016)$ y escolaridad $(p=0.003)$ (Tabla 1$)$. El sector norte de la ciudad tiene un mayor porcentaje de personas con edad igual o superior a 80 años $(15,3 \%)$ en comparación con el sector oeste y sur $(6,6 \%$ y $5,3 \%$ respectivamente). Adicionalmente en el sector norte hay menos adultos mayores con enseñanza supe- rior $(5 \%)$ que en los otros sectores (Oeste: 11,2\%; Este: 7,7\% y Sur: 8,2\%).

En relación al perfil de salud, historial de caídas y actividad física de los adultos mayores de las cuatro regiones geográficas de la ciudad (Tabla 2), se encontraron diferencias en la percepción de salud ( $\mathrm{p}=0.009)$, utilización de medicamentos $(\mathrm{p}=0.001)$ y el nivel de actividad física $(p=0.001)$. La prevalencia del uso de más de dos medicamentos es mayor en el sector oeste $(51,0 \%)$ y sur $(52,0 \%)$ que en los otros sectores (norte: $33,0 \%$ y oeste: $34,0 \%)$.

Según los resultados de la regresión logística multinomial (Tabla 3), se observa que tanto en el análisis crudo como en el análisis ajustado hubo una asociación significativa $(\mathrm{p}<0.05)$ de la región geográfica con todas las variables, excepto el historial de caídas en los últimos seis meses que no mostraron significancia ( $\mathrm{p}>$ 0.05). En el análisis ajustado (Tabla 3), se destaca que los ancianos entre 60 - 69 y 70 - 79 años tenían aproximadamente tres veces más probabilidades de vivir en las regiones sur y oeste, respectivamente, en comparación con los ancianos de 80 años o más. Con respecto a la educación, las personas mayores con educación superior tienen más probabilidades de vivir en el este y oeste en comparación con las personas mayores con educación primaria incompleta. Las personas mayores con mala percepción de salud tenían un 58\% más de probabilidades de vivir en la región oriental en comparación con las personas mayores con buena percepción de salud, así como las personas mayores que informaron tomar más de dos medicamentos tenían más del $50,0 \%$ de probabilidades de vivir en la región oriental. y al sur en comparación con los ancianos que informaron tomar uno o dos medicamentos.

Los adultos mayores presentaron diferencias significativas en todas las variables relacionadas con actividad física entre los diferentes sectores geográficos (Tabla 4). Los adultos mayores del sector norte realizan menos tiempo de actividad física moreda y vigorosa en comparación con los otros grupos $(\mathrm{p}=0.001)$. En relación al comportamiento sedentario los adultos mayores de la región sur presentan menor tiempo sentado durante la semana y durante los finales de semana $(p=0.001)$.

La funcionalidad entre los sectores geográficos (tabla 5) presentó diferencias entre los dominios: comprensión y comunicación, participación social y la funcionalidad total $(p=0.001)$. Mostrando que los adultos mayores de la región norte presentaban mejor funcionalidad que los otros sectores. 
Tabla 1 - Perfil sociodemográfico de los adultos mayores de acuerdo con la región geográfica. 2016, Maringá, Brasil (n = 654).

\begin{tabular}{|c|c|c|c|c|c|c|}
\hline \multirow{3}{*}{ Variables } & \multicolumn{4}{|c|}{ Región geográfica } & \multirow{3}{*}{$x^{2}$} & \multirow{3}{*}{$\mathrm{p}$} \\
\hline & Norte & Oeste & Este & Sur & & \\
\hline & $\mathrm{n}(\%)$ & $\mathrm{n}(\%)$ & $\mathrm{n}(\%)$ & $\mathrm{n}(\%)$ & & \\
\hline \multicolumn{7}{|l|}{ Sexo } \\
\hline Masculino & $110(38,2)$ & $64(22,2)$ & $56(19,4)$ & $58(20,1)$ & \multirow[t]{2}{*}{1,417} & \multirow[t]{2}{*}{0,234} \\
\hline Femenino & $118(32,2)$ & $87(23,8)$ & $86(23,5)$ & $75(20,5)$ & & \\
\hline \multicolumn{7}{|l|}{ Estado civil } \\
\hline Casado & $143(35,7)$ & $94(23,4)$ & $74(18,5)$ & $90(22,4)$ & \multirow[t]{4}{*}{0,698} & \multirow[t]{4}{*}{0,403} \\
\hline Soltero & $24(41,4)$ & $15(25,9)$ & $12(20,7)$ & $7(12,1)$ & & \\
\hline Divorciado/Separado & $31(41,9)$ & $9(12,2)$ & $20(27,0)$ & $14(18,9)$ & & \\
\hline Viudo & $30(24,8)$ & $33(27,3)$ & $36(29,8)$ & $22(18,2)$ & & \\
\hline \multicolumn{7}{|l|}{ Rango etario } \\
\hline 60 a 69 años & $134(34,6)$ & $82(21,2)$ & $76(19,6)$ & $95(24,6)$ & \multirow{3}{*}{5,758} & \multirow{3}{*}{$0,016^{*}$} \\
\hline 70 a 79 años & $59(29,9)$ & $59(29,9)$ & $48(24,4)$ & $31(15,8)$ & & \\
\hline 80 años o mas & $35(50,0)$ & $10(14,3)$ & $18(25,7)$ & $7(10,0)$ & & \\
\hline \multicolumn{7}{|l|}{ Renda mensual } \\
\hline 1 a $2 \mathrm{SM}$ & $149(34,4)$ & $100(23,1)$ & $103(23,8)$ & $81(18,7)$ & \multirow{3}{*}{0,003} & \multirow{3}{*}{0,956} \\
\hline 2,1 a $3 \mathrm{SM}$ & $55(35,3)$ & $40(25,6)$ & $29(18,6)$ & $32(20,5)$ & & \\
\hline Más de 3 SM & $10(33,3)$ & $9(30,0)$ & $3(10,0)$ & $8(26,7)$ & & \\
\hline \multicolumn{7}{|l|}{ Escolaridad } \\
\hline Analfabeto & $34(43,0)$ & $17(21,6)$ & $14(17,7)$ & $14(17,7)$ & \multirow{5}{*}{8,979} & \multirow{5}{*}{$0,003^{*}$} \\
\hline Básica incompleto & $119(42,3)$ & $56(19,9)$ & $43(15,3)$ & $63(22,4)$ & & \\
\hline Básica completo & $40(27,2)$ & $37(25,2)$ & $54(36,7)$ & $16(10,9)$ & & \\
\hline Media completo & $23(24,0)$ & $24(25,0)$ & $20(20,8)$ & $29(30,2)$ & & \\
\hline Superior & $12(23,5)$ & $17(33,3)$ & $11(21,6)$ & $11(21,6)$ & & \\
\hline \multicolumn{7}{|l|}{ Tabaquismo } \\
\hline $\mathrm{Si}$ & $19(29,2)$ & $15(23,1)$ & $22(33,8)$ & $9(13,8)$ & \multirow{3}{*}{0,417} & \multirow{3}{*}{0,518} \\
\hline Nunca fumó & $133(35,9)$ & $91(24,6)$ & $75(20,3)$ & $71(19,2)$ & & \\
\hline No, pero ya fumé & $76(34,7)$ & $45(20,5)$ & $45(20,5)$ & $53(24,2)$ & & \\
\hline \multicolumn{7}{|l|}{ Raza } \\
\hline Blanca & $201(37,9)$ & $118(22,3)$ & $108(20,4)$ & $103(19,4)$ & \multirow{3}{*}{9,303} & \multirow{3}{*}{$0,002^{*}$} \\
\hline Negra & $26(24,8)$ & $28(26,7)$ & $24(22,9)$ & $27(25,7)$ & & \\
\hline Otras & $1(5,3)$ & $5(26,3)$ & $10(52,6)$ & $3(15,8)$ & & \\
\hline \multicolumn{7}{|l|}{ Jubilación ${ }^{\mathrm{a}}$} \\
\hline $\mathrm{Si}$ & $167(34,4)$ & $122(25,2)$ & $102(21,0)$ & $94(19,4)$ & \multirow{2}{*}{0,590} & \multirow{2}{*}{0,442} \\
\hline No & $59(36,4)$ & $28(17,3)$ & $37(22,8)$ & $38(23,5)$ & & \\
\hline
\end{tabular}

*Diferencia significativa (Teste do chi-quadrado); $\mathrm{a}$ = variables con casos ausentes. $\mathrm{SM}=$ Salario Mínimo.

\section{Discusión}

El presente estudio proporciona información sobre las diferencias que existen entre los factores sociodemográficos, raza, nivel educacional, uso de medicamentos, nivel de actividad física, funcionalidad y participación social entre los adultos mayores que viven en diferentes barrios de una comuna. Lo cual podría estar relacionado al estado y autopercepción de salud.

$\mathrm{El}$ envejecimiento es un proceso caracterizado por un descenso en la función física y cognitiva, que puede ser afectada por factores intrínsecos como la prevalencia de algunas enfermedades crónicas y factores extrínsecos asociados a factores sociales como: vivienda, nivel educacional, estado socioeconómico y participación social ${ }^{13,14}$. El estudio demostró que hay diferencias entre las características sociodemográficas, estilos de vida, raza y nivel de actividad física entre los diferentes sectores geográficos. Estas diferencias sociodemográficas podrían estar asociadas a las discrepancias entre los niveles de funcionalidad, percepción de salud y participación social entre las zonas geográficas de la ciudad. En este contexto, estudios han señalado que los factores sociodemográficos son factores determinantes para la salud y la calidad de vida de los adultos mayores ${ }^{15,16}$. 
Tabla 2 - Perfil de salud, historia de caídas y actividad física de los adultos mayores de acuerdo con la región geográfica. 2016, Maringá, Brasil $(\mathrm{n}=654$.

\begin{tabular}{|c|c|c|c|c|c|c|}
\hline \multirow{3}{*}{ Variables } & \multicolumn{4}{|c|}{ Región geográfica } & \multirow{3}{*}{$x^{2}$} & \multirow{3}{*}{$\mathrm{p}$} \\
\hline & Norte & Oeste & Este & Sur & & \\
\hline & n (\%) & $\mathrm{n}(\%)$ & $\mathrm{n}(\%)$ & n (\%) & & \\
\hline \multicolumn{7}{|l|}{ Percepción de salud ${ }^{a}$} \\
\hline Buena & $118(37,3)$ & $92(29,1)$ & $51(16,1)$ & $55(17,4)$ & \multirow{3}{*}{6,906} & \multirow{3}{*}{$0,009^{*}$} \\
\hline Regular & $85(32,4)$ & $49(18,7)$ & $65(24,8)$ & $63(24,0)$ & & \\
\hline Mala & $25(33,8)$ & $10(13,5)$ & $24(32,4)$ & $15(20,3)$ & & \\
\hline \multicolumn{7}{|l|}{ Medicamentos $^{\mathrm{a}}$} \\
\hline Ninguno & $32(33,3)$ & $27(28,1)$ & $20(20,8)$ & $17(17,7)$ & \multirow{3}{*}{10,167} & \multirow{3}{*}{$0,001^{*}$} \\
\hline 1 a 2 & $118(41,4)$ & $72(25,3)$ & $48(16,8)$ & $47(16,5)$ & & \\
\hline Más de 2 & $75(28,0)$ & $52(19,4)$ & $72(26,9)$ & $69(25,7)$ & & \\
\hline \multicolumn{7}{|c|}{ Caídas en los últimos 6 meses $^{\mathrm{a}}$} \\
\hline $\mathrm{Si}$ & $35(33,0)$ & $24(22,6)$ & $21(19,8)$ & $26(24,6)$ & \multirow[t]{2}{*}{0,763} & \multirow[t]{2}{*}{0,382} \\
\hline No & $193(35,3)$ & $127(23,3)$ & $121(22,2)$ & $105(19,2)$ & & \\
\hline \multicolumn{7}{|c|}{ Casi caídas nos últimos 6 meses $^{\mathrm{a}}$} \\
\hline $\mathrm{Si}$ & $33(25,0)$ & $37(28,0)$ & $38(28,8)$ & $24(18,2)$ & \multirow[t]{2}{*}{2,240} & \multirow[t]{2}{*}{0,134} \\
\hline No & $195(37,6)$ & $114(22,0)$ & $103(19,8)$ & $107(20,6)$ & & \\
\hline \multicolumn{7}{|l|}{ Nivel de actividad física } \\
\hline Muy activo/activo & $103(25,9)$ & $94(23,7)$ & $98(24,7)$ & $102(25,7)$ & \multirow{3}{*}{25,565} & \multirow{3}{*}{$0,001^{*}$} \\
\hline Irregularmente activo & $97(53,9)$ & $35(19,4)$ & $26(14,4)$ & $22(12,3)$ & & \\
\hline Sedentario & $28(36,4)$ & $22(28,5)$ & $18(23,4)$ & $9(11,7)$ & & \\
\hline
\end{tabular}

*Diferencia significativa (Teste do chi-quadrado); $a$ = variables con casos ausentes.

Tabla 3 - Análisis de regresión logística multinomial entre la región geográfica (categoría de referencia: norte) y las variables sociodemográficas y de salud. 2016, Maringá, Brasil ( $\mathrm{n}=654)$.

\begin{tabular}{|c|c|c|c|c|c|c|}
\hline \multirow{3}{*}{ Variáveis } & \multicolumn{6}{|c|}{ Región geográfica } \\
\hline & \multicolumn{2}{|c|}{ Oeste } & \multicolumn{2}{|c|}{ Este } & \multicolumn{2}{|c|}{ Sur } \\
\hline & $\mathrm{OR}(\mathrm{IC} 95 \%)^{\dagger}$ & OR (IC 95\%) & $\mathrm{OR}(\mathrm{IC} 95 \%)^{+}$ & OR(IC 95\%) & $\mathrm{OR}(\mathrm{IC} 95 \%)^{+}$ & OR (IC 95\%) \\
\hline \multicolumn{7}{|l|}{ Rango Etario } \\
\hline 60 a 69 años & $2,12[1,00-4,55]^{*}$ & $1,92[0,87-4,28]$ & $1,10[0,58-2,08]$ & $0,78[0,37-1,63]$ & $3,55[1,51-8,32]^{*}$ & $2,69[1,07-6,72]^{*}$ \\
\hline 70 a 79 años & $3,50[1,58-7,71]^{*}$ & $3,45[1,52-7,86]^{*}$ & $1,58[0,80-3,14]$ & $1,31[0,60-2,83]$ & $2,63[1,05-6,60]^{*}$ & $2,31[0,87-6,22]$ \\
\hline 80 años o mas & 1 & 1 & 1 & 1 & 1 & 1 \\
\hline \multicolumn{7}{|l|}{ Escolaridad } \\
\hline Analfabeto & $0,35[0,14-0,90]^{*}$ & $0,42[0,15-1,17]$ & $0,45[0,16-1,26]$ & $0,44[0,14-1,38]$ & $0,45[0,16-1,26]$ & $0,62[0,20-1,91]$ \\
\hline Básica incompleto & $0,33[0,15-0,74]^{*}$ & $0,33[0,14-0,79]^{*}$ & $0,39[0,16-0,96]^{*}$ & $0,34[0,13-0,91]^{*}$ & $0,58[0,24-1,38]$ & $0,55[0,21-1,43]$ \\
\hline Básica completo & $0,65[0,28-1,55]$ & $0,72[0,29-1,78]$ & $1,47[0,59-3,68]$ & $1,55[0,58-4,17]$ & $0,44[0,16-1,19]$ & $0,50[0,17-1,45]$ \\
\hline Media completo & $0,74[0,29-1,88]$ & $0,77[0,29-2,04]$ & $0,95[0,34-2,62]$ & $1,05[0,35-2,13]$ & $1,38[0,51-3,68]$ & $1,49[0,52-4,24]$ \\
\hline Superior & 1 & 1 & 1 & 1 & 1 & 1 \\
\hline \multicolumn{7}{|l|}{ Percepción de salud } \\
\hline Buena & $1,95[0,89-4,26]$ & $2,15[0,91-5,08]$ & $0,45[0,24-0,86]^{*}$ & $0,42[0,19-0,91]^{*}$ & $0,78[0,38-1,59]$ & $0,99[0,44-2,25]$ \\
\hline Regular & $1,44[0,64-3,25]$ & $1,53[0,64-3,66]$ & $0,80[0,42-1,52]$ & $0,75[0,36-1,58]$ & $1,23[0,60-2,53]$ & $1,40[0,63-3,13]$ \\
\hline Mala & 1 & 1 & 1 & 1 & 1 & 1 \\
\hline \multicolumn{7}{|l|}{ Medicamentos } \\
\hline Ninguno & $1,22[0,65-2,27]$ & $0,82[0,41-1,65]$ & $0,65[0,34-1,24]$ & $0,51[0,24-1,08]$ & $0,58[0,30-1,13]$ & $0,49[0,23-1,06]$ \\
\hline 1 a 2 & $0,88[0,56-1,39]$ & $0,74[0,45-1,23]$ & $0,42[0,27-0,68]^{*}$ & $0,44[0,26-0,74]^{*}$ & $0,43[0,27-0,69]$ & $0,43[0,26-0,73]^{*}$ \\
\hline Más de 2 & 1 & 1 & 1 & 1 & 1 & 1 \\
\hline \multicolumn{7}{|c|}{ Casi caídas nos últimos 6 meses } \\
\hline $\mathrm{Si}$ & $1,04[0,59-1,84]$ & $1,32[0,71-2,44]$ & $0,96[0,53-1,72]$ & $0,82[0,42-1,61]$ & $1,37[0,78-2,39]$ & $1,56[0,83-2,92]$ \\
\hline No & 1 & 1 & 1 & 1 & 1 & 1 \\
\hline \multicolumn{7}{|c|}{ Nivel de actividad física } \\
\hline Muy activo/activo & $1,16[0,62-2,17]$ & $1,092[0,57-2,11]$ & $1,48[0,77-2,85]$ & $1,82[0,89-2,74]$ & $3,08[1,39-6,85]^{*}$ & $3,30[1,43-7,60]^{*}$ \\
\hline Irreg. activo & $0,46[0,23-0,91]^{*}$ & $0,46[0,23-0,95]^{*}$ & $0,42[0,20-0,87]^{*}$ & $0,54[0,25-1,20]$ & $0,71[0,29-1,71]$ & $0,76[0,30-1,91]$ \\
\hline Sedentario & 1 & 1 & 1 & 1 & 1 & 1 \\
\hline
\end{tabular}

$\mathrm{OR}=$ odds ratio; $\mathrm{CI}=$ intervalo de confianza; $†$ Análisis crudo; †† Análisis ajustado para todas las variables académicas; ${ }^{*} \mathrm{p}<0.05=\mathrm{Regre-}$ sión logística multinomial. 
Tabla 4 - Comparación de practica de actividad física entre los adultos mayores de acuerdo con la región geográfica. 2016, Maringá, Brasil (n $=654)$.

\begin{tabular}{|c|c|c|c|c|c|}
\hline \multirow{3}{*}{ Variables } & \multicolumn{4}{|c|}{ Región geográfica } & \multirow{3}{*}{$\mathrm{p}$} \\
\hline & 1. Norte & 2. Oeste & 3. Este & 4. Sur & \\
\hline & $\mathrm{Md}(\mathrm{Q} 1 ; \mathrm{Q} 3)$ & $\mathrm{Md}(\mathrm{Q} 1 ; \mathrm{Q} 3)$ & $\mathrm{Md}(\mathrm{Q} 1 ; \mathrm{Q} 3)$ & $\mathrm{Md}(\mathrm{Q} 1 ; \mathrm{Q} 3)$ & \\
\hline Días de caminata & $5,0(3,0 ; 7,0)$ & $3,0(1,0 ; 5,0)$ & $2,5(2,0 ; .5,0)^{\mathrm{a}}$ & $5,0(2,5 ; 7,0)$ & $0,001^{*}$ \\
\hline Min. de caminata $\mathrm{p} /$ día & $40,0(16,3 ; 120,0)$ & $30,0(10,0 ; 45,0)^{\mathrm{b}}$ & $60,0(30,0 ; 82,5)$ & $60,0(27,5 ; 180,0)$ & $0,001^{*}$ \\
\hline Min. de caminata $\mathrm{p} / \mathrm{sem}$. & $180,0(62,5 ; 810,0)$ & $80,0(15,0 ; 180,0)^{c}$ & $140,0(60,0 ; 272,5)$ & $180,0(85,0 ; 660,0)$ & $0,001^{*}$ \\
\hline Días Ativ. moderada & $0,0(0,0 ; 2,0)^{\mathrm{d}}$ & $2,0(0,0 ; 3,0)$ & $2,0(0,0 ; 3,0)$ & $2,0(0,0 ; 4,0)$ & $0,001^{*}$ \\
\hline Min. Ativ. moderada p/ dia & $0,0(0,0 ; 30,0)^{e}$ & $20,0(0,0 ; 60,0)$ & $60,0(0,0 ; 120,0)$ & $40,0(0,0 ; 160,0)$ & $0,001^{*}$ \\
\hline Min. Atv. moderada p/ sem. & $0,0(0,0 ; 120,0)^{f}$ & $30,0(0,0 ; 180,0)$ & $90,0(0,0 ; 240,0)$ & $100,0(0,0 ; 180,0)$ & $0,001^{*}$ \\
\hline Días de atividade vigorosa & $0,0(0,0 ; 0,0)^{\mathrm{g}}$ & $0,0(0,0 ; 1,0)$ & $0,0(0,0 ; 1,0)$ & $0,0(0,0 ; 1,0)$ & $0,001^{*}$ \\
\hline Min. Atv. vigorosa $\mathrm{p} / \mathrm{dia}$ & $0,0(0,0 ; 0,0)^{\mathrm{h}}$ & $0,0(0,0 ; 45,0)$ & $0,0(0,0 ; 60,0)$ & $0,0(0,0 ; 12,5)$ & $0,001^{*}$ \\
\hline Min. Atv. vigorosa $\mathrm{p} / \mathrm{sem}$. & $0,0(0,0 ; 0,0)^{\mathrm{i}}$ & $0,0(0,0 ; 60,0)$ & $0,0(0,0 ; 120,0)$ & $0,0(0,0 ; 30,0)$ & $0,001^{*}$ \\
\hline Tiempo sentado durante sem. & $240,0(120,0 ; 345,0)$ & $330,0(240,0 ; 480,0)$ & $300,0(142,5 ; 420,0)$ & $180,0(120,0 ; 240,0)^{\mathrm{j}}$ & $0,001^{*}$ \\
\hline Tiempo sentado fin de sem. & $240,0(120,0 ; 360,0)$ & $480,0(300,0 ; 840,0)$ & $360,0(262,5 ; 480,0)$ & $240,0(120,0 ; 360,0)^{k}$ & $0,001^{*}$ \\
\hline
\end{tabular}

*Diferencia significativa $=p<0,05-$ Test de Kruskal-Wallis seguido del test "U" de Mann-Whitney entre = a) 3 con 1 e 4; b) 2 con 1, 3 e 4; c) 2 con 1,3 e 4 ; d, e, f, g, h, i) 1 con 2,3 e 4 ; j, k) 4 con 1,2 e 3 .

Tabla 5 - Comparación de la funcionalidad entre los adultos mayores de acuerdo con la región geográfica. 2016, Maringá, Brasil (n = 654).

\begin{tabular}{|c|c|c|c|c|c|}
\hline \multirow{3}{*}{ Variables } & \multicolumn{4}{|c|}{ Región geográfica } & \multirow{3}{*}{$\mathrm{p}$} \\
\hline & 1. Norte & 2. Oeste & 3. Este & 4. Sur & \\
\hline & Md (Q1; Q3) & $\mathrm{Md}(\mathrm{Q} 1 ;$ Q3) & Md (Q1; Q3) & Md (Q1; Q3) & \\
\hline Comprensión y comunicación & $0,0(0,0 ; 1,0)^{a}$ & $1,0(0,0 ; 2,0)$ & $1,0(0,0 ; 2,0)$ & $1,0(0,0 ; 2,0)$ & $0,001^{*}$ \\
\hline Movilidad & $0,0(0,0 ; 3,0)^{\mathrm{b}}$ & $2,0(0,0 ; 3,0)$ & $2,0(1,0 ; 3,0)$ & $1,0(0,0 ; 2,0)$ & $0,001^{*}$ \\
\hline Auto-cuidado & $0,0(0,0 ; 0,0)$ & $0,0(0,0 ; 0,0)$ & $0,0(0,0 ; 0,0)$ & $0,0(0,0 ; 0,0)$ & 0,975 \\
\hline Relaciones interpersonales & $0,0(0,0 ; 0,0)$ & $0,0(0,0 ; 0,0)$ & $0,0(0,0 ; 1,0)$ & $0,0(0,0 ; 1,0)$ & 0,456 \\
\hline Atividades de la vida diaria & $0,0(0,0 ; 2,0)^{c}$ & $1,0(0,0 ; 2,0)$ & $1,0(0,0 ; 2,0)$ & $1,0(, 0 ; 2,0)$ & $0,001^{*}$ \\
\hline Participación social & $0,0(0,0 ; 1,0)^{\mathrm{d}}$ & $1,0(0,0 ; 2,0)$ & $2,0(0,0 ; 3,0)$ & $1,0(0,0 ; 2,5)$ & $0,001^{*}$ \\
\hline Funcionalidad total & $2,0(0,0 ; 5,0)^{\mathrm{e}}$ & $6,0(1,0 ; 11,0)$ & $7,0(3,8 ; 10,0)$ & $5,0(0,0 ; 9,0)$ & $0,001^{*}$ \\
\hline
\end{tabular}

*Diferencia significativa $=\mathrm{p}<0,05-$ Test de Kruskal-Wallis seguido del test "U" de Mann-Whitney entre = a, b, c, d, e) 1 con 2, 3, e 4.

Nuestro estudio ha reportado que aquellos individuos de sectores que tienen mejores niveles de educación y mejores salarios son también los que presentan una mejor percepción de la salud y un mayor nivel de actividad física, lo que puede estar directamente relacionado a su menor consumo de medicamentos observado en estos sectores. Estos hallazgos son similares a los publicados por otros autores ${ }^{17,18}$. Estudios recientes en América Latina han observado que un buen nivel educacional y económico está altamente relacionado con mayor funcionalidad, salud mental, menor discapacidad y prevalencia de enfermedades y con un alto nivel de participación social principalmente en adultos mayores ${ }^{17,19,20}$.

La Organización Mundial de la Salud por medio de la Clasificación Internacional de Discapacidad y Funcionalidad (CIF) describe una estrecha relación entre las funciones físicas con el nivel de autonomía y de participación en la comunidad ${ }^{8}$. Que está asociado tanto a factores intrínsecos de la persona, como a la motivación y la propia percepción de la salud, así como a factores ambientales relacionados principalmente a la vivienda y ubicación geografía ${ }^{8}$. Estos factores contextuales pueden influenciar en el estado de salud del individuo.

En el presente estudio encontramos diferencias entre las percepciones de salud de las localidades, así como en la utilización de medicamentos. Las regiones que describen en un mayor porcentaje su salud como mala (Este: 17,1\% y Sur: 11,2\%), son también las que consumen más de dos tipos de medicamentos $(51,0 \%$ y $51,8 \%$ respectivamente). En esta línea, Sacchi, Hausberger, y Pereyra ${ }^{22}$ señalan que la percepción de los problemas de salud está ligada a condiciones materiales de vida y a factores socioculturales ${ }^{22}$. La misma autora sugiere que es necesario hacer análisis socioculturales para poder comprender la relación de la población con el Sistema de Salud ${ }^{22}$. De este modo, nuestro estudio 
reafirma que dependiendo del barrio donde vive el adulto mayor, puede haber importantes variables que modifican los indicadores de salud. Por lo que las estrategias para abordar las temáticas de Salud, en el sistema público no se pueden generalizar para toda una población, sino que deben ser estudiadas de acuerdo con las condiciones de cada localidad ${ }^{18}$. Cada sector tiene sus propias características, incluso en una misma ciudad, las cuales están relacionadas al nivel de escolaridad, renta mensual, edad y distribución de género. En este sentido, la literatura sugiere considerar estos factores contextuales para que una estrategia de salud tenga éxito y la población tenga una participación activa y se sienta comprometido con su salud.

Otro aspecto también evaluado fue el nivel actividad física, observándose diferencias entre los niveles de actividad física entre los sectores. A pesar de no haber presentado diferencias entre el género entre los adultos mayores de los barrios, se observaron diferencias en los niveles de actividad física. De acuerdo con la literatura hay diferentes factores asociados a la actividad física como son la capacidad funcional, participación social, nivel educacional y zona geografica ${ }^{23,24}$. La participación social definida como la participación activa en organizaciones comunitarias de carácter religioso, deportivo, cultural, recreativos, políticas y de voluntariados ${ }^{25-27}$ podría tener una estrecha relación con las condiciones de salud, nivel de actividad física y funcionalidad. Diversos estudios han reportado efectos protectores de la participación social para la salud, siendo considerada como un estímulo para aumentar el nivel de actividad física y la capacidad de sociabilización, favoreciendo las funciones cognitivas ${ }^{25,28}$. Uno de los factores relevantes que se ha asociado con la participación social de los adultos mayores es el nivel educacional, el cual presentó diferencias entre los diferentes barrios en nuestro estudio. En este sentido, más años de escolaridad han sido asociados con mayor funcionalidad y aumentos de la participación social en adultos mayores $^{29,30}$. Adicionalmente, la zona geográfica también es un factor importante para la participación social y la práctica de la actividad física, de acuerdo con Bauman y colaboradores individuos que viven en barrios con mayores áreas recreativas, parques, áreas verdes o cerca de la playa tienen un mayor nivel de actividad física. De este modo, la zona geográfica y sus propios factores contextuales ya mencionados anteriormente, son determinantes para la salud física de la población ${ }^{31}$.

A pesar de los resultados encontrados en el estudio, nuestro trabajado presenta algunas limitaciones. No realizamos evaluaciones como test funcionales o con monitores de actividad física. Sin embargo, utilizamos cuestionarios validados, que tienen una alta aplicabilidad para la realidad clínica. Además, realizamos una muestra representativa de la población de la comuna con aleatorización de los centros de sistema de salud para ser evaluados. Donde conseguimos abordar, la mayoría de los centros de atención primaria de la cuidad. Futuros estudios, idealmente con un diseño longitudinal, podrían evaluar el impacto de los factores socioculturales, demográficos, funcionales y biológicos en la condición de salud, y cómo los de adultos mayores responden a las intervenciones de salud dependiendo del barrio y los otros factores contextuales.

Existen diferencias entre los factores sociodemográficos, raza, nivel educacional, uso de medicamentos, nivel de actividad física, funcionalidad y participación social entre los adultos mayores que viven en diferentes barrios de una comuna que podrían influenciar la condición de salud. Estas diferencias, deben ser consideradas al momento de crear estrategias de salud pública en los diferentes sectores, debido a que cuentan con características y necesidades específicas.

\section{Conflicto de interese}

Los autores declaran no tener ningún conflicto de intereses.

\section{Contribuciones de los autores}

Oliveira DV y Loyola WAS participaron en el diseño inicial del estudio, la redacción y la revisión crítica del texto. Freire GLM, Maciel RPT y Kerber VL fueron responsables de buscar en la literatura y recopilar datos. Nascimento Júnior JRA participó en la revisión crítica del texto y el análisis de datos.

\section{Referencias}

1. Canêdo AC, Lopes CS, Lourenço RA. Prevalence of and factors associated with successful aging in Brazilian older adults: Frailty in Brazilian older people Study (FIBRA RJ). Geriatr Gerontolo Int. 2018;18(8):1280-5.

2. Kingston A, Robinson L, Booth H, Knapp M, Jagger C. Projections of multi-morbidity in the older population in England to 2035: estimates from the Population Ageing and Care Simulation (PACSim) model. Age Ageing. 2018;47(3):374-80.

3. Ramachandran R, Mundodan JM, Saju C, Joshy VM. Nutritional status and cognitive impairment in elderly population in a rural area of Thrissur district, Kerala. IJCMPH. 2018;5(3):1218-23.

4. Fechine BRA, Trompieri N. O processo de envelhecimento: as principais alterações que acontecem com o idoso com o passar dos anos. Int Sci Place. 2015;1(20):106-32. 
5. Abedzadeh-Kalahroudi M, Razi E, Sehat M. The relationship between socioeconomicstatus and trauma outcomes. J Public Health. 2018;40(4):e431-e439.

6. Bossuyt N, Gadeyne S, Deboosere P, Van Oyen, H. Socioeconomic inequalities in health expectancy in Belgium. Public Health. 2004;118(1):3-10.

7. Szwarcwald CL, Montilla DER, Marques AP, Damacena GN, Almeida WS, Malta DC. Inequalities in healthy life expectancy by Federated States. Rev Saude Pública. 2017;51 (suppl1): $7 \mathrm{~s}$.

8. Organization, W. H. Healthy cities and urban governance. Geneva, Switzerland: World Health Organization. 2006.

9. Brucki SM, Nitrini R, Caramelli P, Bertolucci PH, Okamoto IH. Sugestões para o uso do mini-exame do estado mental no Brasil. Arq Neuro-Psiquiatr. 2003;61(3B):777-81.

10. Üstün TB, Chatterji S, Kostanjsek N, Rehm J, Kennedy C, Epping-Jordan J, Pull C. Developing the World Health Organization disability assessment schedule 2.0. Bulletin WHO 2010;88:815-23.

11. Moreira A, Alvarelhão J, Silva AG, Costa R, Queirós A. Tradução e validação para português do WHODAS 2.0: 12 itens em pessoas com 55 ou mais anos. Rev Port Saúde Pública. 2015;33(2):179-82.

12. Benedetti TRB, Antunes PC, Rodriguez-Añez CR, Mazo GZ, Petroski ÉL. Reproducibility and validity of the International Physical Activity Questionnaire (IPAQ) in elderly men. Rev Bras Med Esp. 2007;13(1):11-6.

13. Cruz-Jentoft AJ, Baeyens JP, Bauer JM, Boirie Y, Cederholm T,Landi F, et al. Sarcopenia: European consensus on definition and diagnosis: Report of the European Working Group on Sarcopenia in Older People. Age Aging. 2010;39(4):412-23.

14. Tuna HD, Edeer AO, Malkoc M, Aksakoglu G. Effect of age and physical activity level on functional fitness in older adults. Eur Rev Aging Phys Act. 2009;6(2):99-106.

15. Takagi D, Kondo K, Kawachi I. Social participation and mental health: moderating effects of gender, social role and rurality. BMC Public Health. 2013;13(1):701-9.

16. Willie-Tyndale D, Holder-Nevins D, Mitchell-Fearon K, James K, Laws H, Waldron NK, et al. Participation in social activities and the association with sociodemographic and health-related factors among community-dwelling older adults in Jamaica. J Cross Cult Gerontol. 2016;31(4):427-47.7

17. Gomes GC, Teixeira-Salmela LF, Fonseca BE, Freitas FAZ, Fonseca MLM, Pacheco BD, et al. Age and education influence the performance of elderly women on the dual-task Timed Up and Go test. Arq Neuropsiquiatr. 2015;73(3):187-93.

18. Kendall GE, Nguyen H, Ong R. The association between income, wealth, economic security perception, and health: a longitudinal Australian study. Heal Sociol Rev. 2019;28(1):20-38.

19. Sepúlveda-Loyola W, Ganz F, Maciel RPT, Lopes RDS, Negri PS, Solorza EM, et al. Social participation is associated with better functionality, health status and educational level in elderly women. Brazilian J Dev. 2020;5(6):5983-92.
20. Sepúlveda-loyola W, Rosielma Lopes DS, Maciel RPT, Probst VS. Artículo de revisión participación social, un factor a considerar en la evaluación clínica del adulto mayor: una revisión narrativa. Rev Peru Med Exp Salud Publica. 2020;37(2):341-49.

21. Howard-Grabman L, Miltenburg AS, Marston C, Portela A. Factors affecting effective community participation in maternal and newborn health programme planning, implementation and quality of care interventions. BMC Pregnancy Childbirth. 2017;17(1):1-18.

22. Sacchi M, Hausberger M, Pereyra A. Percepción del proceso salud-enfermedad-atención y aspectos que influyen en la baja utilización del Sistema de Salud, en familias pobres de la ciudad de Salta. Salud colectiva. 2007;3:271-83.

23. Quino-Ávila AC, Chacón-Serna MJ. Capacidad funcional relacionada con actividad física del adulto mayor en Tunja, Colombias. Horiz sanitario. 2018;17(1):59-68.

24. Warren M, Ganley KJ, Pohl PS. The association between social participation and lower extremity muscle strength, balance, and gait speed in US adults. Prev Med Rep. 2016; 4:142-7.

25. Loyola WS, Camillo CA, Torres CV, Probst VS. Effects of an exercise model based on functional circuits in an older population with different levels of social participation. Geriatr Gerontol Int. 2018;18(2):216-23.

26. Pollack CE, Von dem Knesebeck O. Social capital and health among the aged: comparisons between the United States and Germany. Health Place. 2004;10(4):383-91.

27. Sirven N, Debrand T. Social participation and healthy ageing: an international comparison using SHARE data. Soc Sci Med. 2008;67(12):2017-26.

28. Douglas H, Georgiou A, Westbrook J. Social participation as an indicator of successful aging: an overview of concepts and their associations with health. Aust Health Rev. 2017;41(4):455-62.

29. Pinto JM, Neri AL. Factors related to low social participation in older adults: findings from the Fibra study, Brazil. Cad saúde colet. 2017;25(3):286-93.

30. Wamser EL, Valderramas SR, Schieferdecker MEM, Amarante TP, Coelho RA, Guimarã ATB, Gomes ARS. Melhor desempenho no teste timed up and go está associado a melhor desempenho funcional em idosas da comunidade. Geriatr Gerontol Aging. 2015;9(4):138-43.

31. Sohn EK, Porch T, Hill S, Thorpe Junior R. Geography, Race/ Ethnicity, and Physical Activity Among Men in the United States. Am J Mens Health. 2017;11(4):1019-27.

Recibido: $18 / 03 / 2020$

Aprobado: $17 / 07 / 2020$

Cómo citar el artículo:

Oliveira DV, Loyola WAS, Freire GLM, Maciel RPT, Kerber VL, Nascimento Júnior JRA. Actividad física, salud y funcionalidad entre adultos mayores según la localidad geográfica. Rev Bras Ativ Fis Saúde. 2019;24:e0109. DOI: 10.12820/rbafs.24e0109 